\title{
BMJ Open Are predominantly western standards and expectations of informed consent in surgery applicable to all? A qualitative study in a tertiary care hospital in Sri Lanka
}

\author{
Udagedara Mudiyanselage Jayami Eshana Samaranayake, ${ }^{1}$ \\ Yasith Mathangasinghe, ${ }^{1}$ Anura Sarath Kumara Banagala ${ }^{2}$
}

To cite: Samaranayake UMJE, Mathangasinghe $\mathrm{Y}$, Banagala ASK. Are predominantly western standards and expectations of informed consent in surgery applicable to all? A qualitative study in a tertiary care hospital in Sri Lanka. BMJ Open 2019;9:e025299. doi:10.1136/ bmjopen-2018-025299

- Prepublication history and additional material for this paper are available online. To view these files, please visit the journal online (http://dx.doi org/10.1136/bmjopen-2018025299).

Received 12 July 2018 Revised 28 November 2018 Accepted 28 November 2018

D Check for updates

(c) Author(s) (or their employer(s)) 2019. Re-use permitted under CC BY-NC. No commercial re-use. See rights and permissions. Published by BMJ.

${ }^{1}$ Department of Anatomy, Faculty of Medicine, University of Colombo, Colombo, Sri Lanka ${ }^{2}$ National Hospital of Sri Lanka, Colombo, Sri Lanka

\section{Correspondence to} Dr Udagedara Mudiyanselage Jayami Eshana Samaranayake; eshana_sam@live.com.sg

\section{ABSTRACT}

Objective To identify the different perceptions on informed surgical consent in a group of Sri Lankan patients.

Methods A qualitative study was conducted in a single surgical unit at a tertiary care hospital from January to May 2018. The protocol conformed to the Declaration of Helsinki. Patients undergoing elective major surgeries were recruited using initial purposive and later theoretical sampling. In-depth interviews were conducted in their native language based on the grounded theory. Initial codes were generated after analysing the transcripts. Constant comparative method was employed during intermediate and advanced coding. Data collection and analyses were conducted simultaneously, until the saturation of the themes. Finally, advanced coding was used for theoretical integrations.

Results Thirty patients (male:female $=12: 18$ ) were assessed. The mean age was $41 \pm 9$ years. Sinhalese predominated $(50.0 \%, n=15)$. Majority underwent thyroidectomy $(36.7 \%, n=11)$. The generated theory categorises the process of obtaining informed consent in four phases: initial interaction phase, reasoning phase, convincing phase and decision-making phase. Giving consent for surgery was a dependent role between patient, family members and the surgeon, as opposed to an individual decision by the patient. Some patients abstained from asking questions from doctors since doctors were 'busy', 'short-tempered' or 'stressed out'. Some found nurses to be more approachable than doctors. Patients admitted that having a bystander while obtaining consent would relieve their stress. They needed doctors to emphasise more on postoperative lifestyle changes and preprocedure counselling at the clinic level. To educate patients about their procedure, some suggested leaflets or booklets to be distributed at the clinic before ward admission. The majority disliked watching educational videos because they were 'scared' to look at surgical dissections and blood.

Conclusion The informed consent process should include key elements that are non-culture specific along with elements or practices that consider the cultural norms of the society.

\section{Strengths and limitations of this study}

- First study conducted in Sri Lanka addressing the perceptions in surgical consent taking.

- A qualitative approach was used to explore and gather information on the themes until saturation rather than assessing perceptions on a set of questions.

- This study is not a representation of the entire population due to the limitation of the sample size of 30 and a single-centre study. But the findings of this study can be used in hypothesis generation and the design of future studies.

- Attitudes or views of private sector hospital patients should be studied in the future.

\section{INTRODUCTION}

Informed surgical consent (ISC) is for the protection of the patient rights while legally safeguarding the surgical personnel. ${ }^{1}$ Surgeons are obliged to avoid any unnecessary interventions and haphazard surgical procedures. ${ }^{1}$ Ethically it provides patients self-governance in their decision, shifting the surgical practice to a 'patient-centred' approach. ${ }^{1}$ Documentation in ISC is to ensure the complete understanding of the surgical procedure by the patient and confirmation of the agreement with surgeons to perform interventions on their bodies. ${ }^{2}$

Inadequacies in ISC that may affect the ethical and legal liberties of the patients are reported nationally and internationally. Grauberger et al in an American study on ISC in spinal surgery indicated a failure in consent in $66 \%$ of procedures, out of which majority were due to the lack of explanation of adverse effects of surgery $(30.4 \%)$ and failure in giving alternative treatment options $(9.9 \%) .{ }^{3}$ A British study was conducted to test 
recollection of information 1 day after consent form was signed. This concluded that only $60 \%$ understood the nature and purpose of the surgery with only $55 \%$ being able to state least one major complication. ${ }^{4}$ A Sri Lankan study by Wijerathne et al on parent's knowledge on their child's surgery in Lady Ridgeway Hospital for Children concluded that the vital information about the surgery was not known by the parents regardless of their educational status. ${ }^{5}$ Therefore, it is apparent that lack in ISC is an issue that needs to be addressed all around the world.

ISC is a concept that keeps evolving and changing. Due to the legal implications, in most of the developed countries it is more of a protocol-based approach. ${ }^{6}$ However, there is recent evidence to suggest a shift in the western countries' attitude towards a shared decision-making practice between the patient and the clinician through a landmark supreme court ruling 'The Montgomery case (2017)' in the UK. ${ }^{7}$ Beauchamp and Childress mentioned that one of the four principles in healthcare ethics is to respect patient autonomy. ${ }^{8}$ Patient's capacities and perceptions should be entertained while avoiding manipulative under-revelation of key information to establish a self-chosen method of management. ${ }^{8}$ Nevertheless, ethical judgements of patients widely vary depending on individual values.

Expectations of patients might differ according to cultural norms. ${ }^{9}$ Sri Lanka is a developing country in South Asia, which is home to more than 20 million people from diverse ethnic and religious backgrounds. There were no previous studies done in Sri Lanka discussing how the patients perceived the idea of ISC. The objective of this study was to identify the extent to which our patients wish the details of their surgery to be disclosed and what changes are required in our surgical setting to address their needs. We also intended to study and compare the differences in the level of authority used in our consent-taking protocol, as compared with the international guidelines.

\section{METHOD}

This qualitative study was conducted at a single surgical unit in a tertiary care teaching hospital in Sri Lanka from January to May 2018.

\section{Ethics}

The protocol of the study conformed to the Declaration of Helsinki. Approval was obtained from the hospital ethical clearance committee (reference no ETH/ $\mathrm{COM} / 2018 / 03$ ) and the patients before the commencement of the interviews. Informed written consent was obtained in their own language from all the participants before the interview. The purpose, risks and benefits of the research were explained to the participants by the principal investigator prior to obtaining the consent. All participants were informed that they had the right to refuse consent or withdraw from the study at any time without having any impact on the healthcare provided to them. Patients had the right to remain silent if they did not wish to answer any question. Patient identifiers were not used to maintain anonymity.

\section{Participant involvement}

The research question we studied was based on our observations regarding the gaps and dissimilarities of obtaining ISC in the local and regional hospitals. Patients did not participate in designing the study or recruiting participants. Patient identifiers were not used due to ethical reasons. Thus, we could not directly communicate the results of the study with the individual patients. But the dissemination plan includes an awareness programme for medical doctors and developing a booklet for patients in light of this research.

\section{Data collection}

This qualitative study was based on the grounded theory. ${ }^{10}$ The sampling universe was defined according to the following criteria. Patients undergoing major elective surgeries were included. Patients below the age of 18 , patients with the evidence of reduced cognitive capacity and of those whom the elective surgeries were postponed after obtaining the ISC on a previous hospital admission were excluded from the study. Since the study population was a vulnerable group, a face-to-face advertising method was employed as the recruitment method. ${ }^{11}$ Once the patients recovered after the surgery and before discharge, they were invited to participate in the study by the principal investigators. After a one-on-one presentation describing the purpose, procedure and the nature of the study, the patients were allowed to clarify their doubts. Sampling strategy involved a mixed method. Initially the patients were recruited using purposive sampling. ${ }^{11}$ Patients from different genders, age categories and ethnic groups (ie, Sinhala, Tamil, Moor, Burgher) were initially targeted to include a heterogeneous stratified sample. Later, a theoretical sampling approach was used to explore new groups and emerging themes with simultaneous analysis of data. ${ }^{13}$ Patients were interviewed by two interviewers. The principal investigator and the interviewers were medical doctors who were independent of the clinical team caring for the patient. Interviewers were not known to any of the patients. Interviews which lasted 45 to $60 \mathrm{~min}$ were conducted in an undisturbed room in the surgical ward. In-depth interviews were conducted in the patient's native language (Sinhalese, Tamil or English). Initially, the patient's views on ISC and alterations in the current system which would help them understand the purpose of their surgery were assessed using open-ended questions. Later, as more themes developed with the data analysis, semistructured questionnaires were used to explore emerging themes. An interview topic guide is given as online supplementary file 1 .

\section{Data analysis}

Interviews were recorded and fully transcribed. The transcriptions were analysed by two authors (YM and 
UMJES) through several readings. A coding framework was employed to analyse data (online supplementary file 2). Open codes were generated using verbatim quotes of the participants. ${ }^{14}$ Then the related codes were categorised together. ${ }^{14}$ Codes and categories were constantly compared between and within groups with the concurrent data collection process. ${ }^{14}$ This was followed by axial coding while developing subcategories and constant comparison of data. ${ }^{14-16}$ Context was modified through deliberations and re-reading of the interviews. Peer debriefing and their evaluations were used to make modifications. Multiple coding in the same dataset was conducted by different investigators to increase the validity of the study. Disagreements between the investigators after multiple coding were reconciled by iterative discussions. Interviews were conducted until the normothermic target was achieved. ${ }^{17}$ Saturation of themes was achieved when new data analysis returned similar codes within the prevailing categories, where the latter adequately explained the generated themes. ${ }^{14}$ Finally, advanced coding was conducted for theoretical integrations. ${ }^{15}$

\section{RESULTS}

The study population comprised 30 patients (male : female $=12: 18)$. Mean age was $41 \pm 9$ years. A major proportion was Sinhalese $(50.0 \%, \mathrm{n}=15)$, followed by Moors $(30.0 \%, \mathrm{n}=9)$, Tamils $(16.7 \%, \mathrm{n}=5)$ and Burghers $(3.3 \%$, $\mathrm{n}=1)$. Majority underwent total thyroidectomy $(36.7 \%$, $\mathrm{n}=11)$ and laparoscopic cholecystectomy $(26.7 \%, \mathrm{n}=8)$. Twenty-four $(80 \%)$ had had at least primary education while $17(56.7 \%)$ had completed their secondary education. Six $(20.0 \%)$ did not have any formal education. Majority (63.3\%, $\mathrm{n}=19$ ) had not undergone previous surgeries. Only two patients declined to participate in the study, hence the positive response rate was $93.8 \%$.

The resultant theory generated in this grounded study categorises the process of obtaining ISC in four phases: initial interaction phase, reasoning phase, convincing phase and decision-making phase (figure 1). The initial interaction phase was where the patient encountered the surgeon for the first time. This commonly happened in the clinic setting. Outcome of the initial interaction phase depended on preprocedural interpretation of consent by the patients and the setting of initial interaction. In the reasoning phase, there were dynamic interactions between the clinician and the patient regarding the rationale for undergoing surgery. Reasoning for the surgery was influenced by the time spent on explanations and individual expectations of the sufficiency of information. The convincing phase was a state of equilibrium between the doctors' views and patients' wishes. It was based on the content of the consent and the family member contribution. The decision-making phase depended on the choice of the patient to undergo or withdraw from the surgery. This was influenced by the professional governance in decision-making and availability of the access to the surgical facilities by the patient.

The concepts of the generated theory are further discussed below.

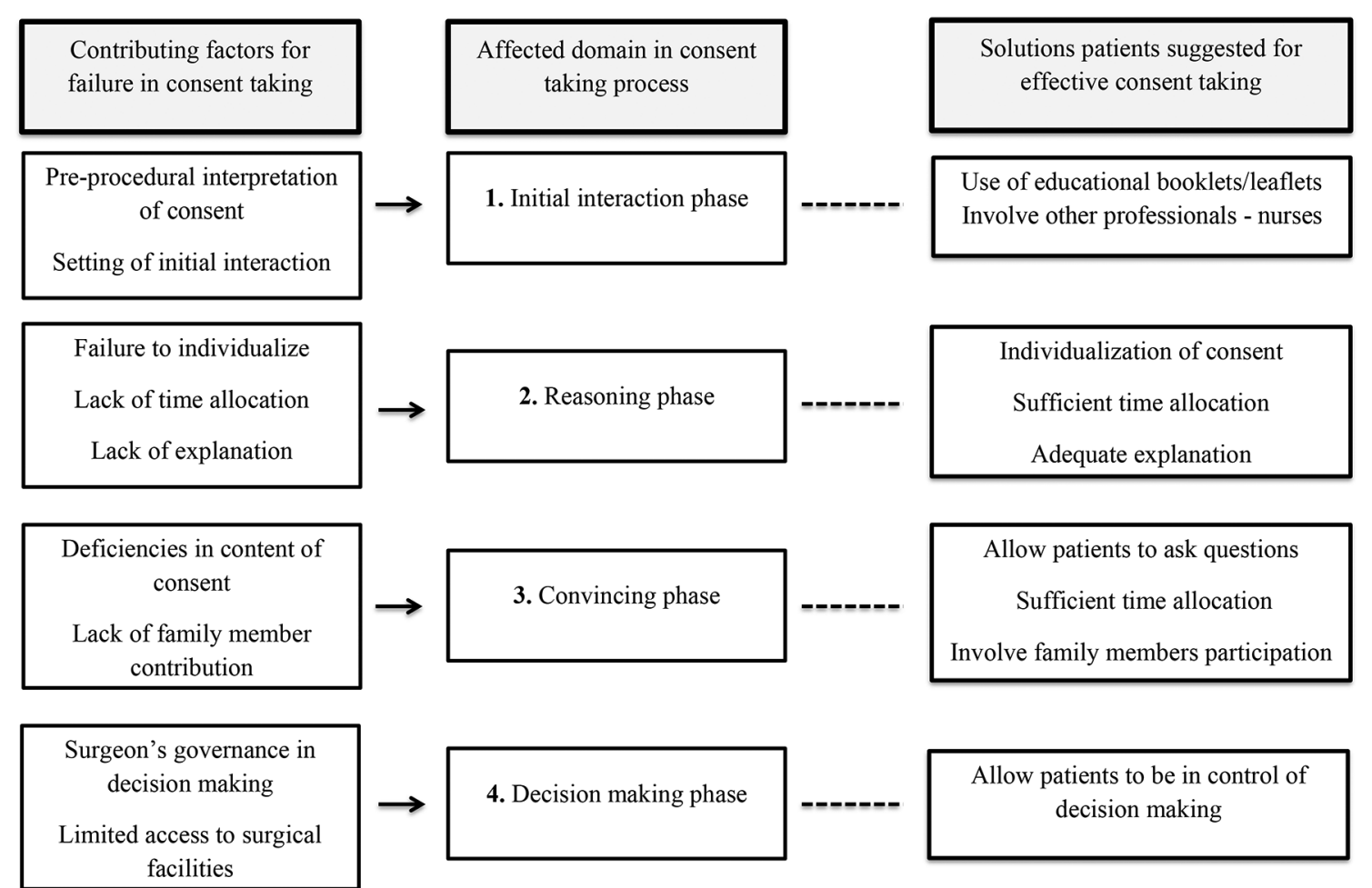

Figure 1 Comparison of key findings in contributing factors, affected domains in consent process and suggestions by patients to overcome the issues. 


\section{What ISC meant to the interviewees}

\section{Professional governance in decision}

Few patients expressed that they were content with what the doctor decided for them. One patient stated 'Decision making is a doctor's job. I have no objections to their opinion' (Interviewee 15, bariatric surgery). This interviewee was not interested in questioning or finding out other surgical options.

Although some patients realised that the ISC was not detailed enough, they did not want to question the doctors out of courtesy. 'Doctors are busy and stressed out, so I feel bad to ask questions from them' (interviewee 6 , anterior resection).

However, majority of the patients did not share this view. In contrast, they wanted to know more about the outcomes of their surgery. This is further discussed in the subsequent sections.

Their lack of insight into surgical care was apparent by 'I don't know about our condition, so we are dependent on the doctor. I don't know who else to question apart from the doctor allocated to me' (Interviewee 26, pancreatectomy).

\section{Legal entity}

Some patients believed that it was their right to information. 'I believe I have the right to know what is being done to my body, so consenting is important' (interviewee 16, mastectomy).

Some found it difficult to reject the surgery and did not consider it to be their legal right to do so. 'We cannot say no, if we do we will be asked to leave. I travelled so far just for this surgery' (interviewee 6 , cholecystectomy).

Few patients felt that the ISC was merely a formal process. They did not necessarily have blind faith in the doctor. They said that the doctors do it to ensure their own legal protection over what is beneficial for the patients. One patient stated, 'Signature in consent taking is all about making sure no harm comes to the doctor. Whatever happens during surgery, it is I who has to suffer' (interviewee 7 , oesophagectomy).

\section{Who should be involved in the consent-taking process}

One patient highlighted the fact that doctors should take the information directly from the patient rather than questioning the bystander. 'Most of the information about me was gathered from my bystanders rather than directly questioning me. So, I thought that the bystander was supposed to consent for me' (interviewee 22, thyroidectomy).

Some mentioned that a family member should be present at the time of explanation. 'It is better if a family member is present when the doctor gives details about the surgery, because we are distressed. So (I) cannot comprehend what's being told' (interviewee 25, cholecystectomy). 'My family will be affected by my decision, (therefore) it is better if they can also give their ideas' (interviewee 4 , thyroidectomy).
Gaps in the informed consent process

Inadequacy of time spent

Inadequacy of time allocation to explain the surgery before admission was not accepted by some patients. They wanted the doctors to spend a suitable amount of time in the clinic to ensure that they were well counselled. In addition, they expected the doctors to provide them the opportunity to clarify their doubts in the clinic rather than in the ward. This was vividly conveyed by the following comment. 'I was not given enough time and information about the operation. So, I consulted a doctor in a private hospital to get more information about my operation' (interviewee 5, cholecystectomy).

Limited time was given to think on and respond to the informed consent. This made a few patients feel that the ISC was a forced practice. 'Doctor said that the ward setting was busy, and she did not have enough time for me. I was asked to sign on a paper' (interviewee 3, thyroidectomy). Thus, they wanted more time to decide and give their final opinion on the surgery in the ward setting.

\section{Deficiency in content of consent}

\section{At the initial encounter}

The common impression was that vague or no explanations about their surgical procedure at the clinic setting caused confusion.

'I was not explained what my surgical procedure was until I was hospitalized for the surgery' (interviewee 5, cholecystectomy).

'I had to ask my relatives and friends who underwent breast cancer treatment about their experiences. Some said they underwent radiotherapy and some didn't. I was confused about what the exact treatment I was supposed to undergo' (interviewee 1, mastectomy).

Majority of the patients wanted a basic idea of their surgery in a clinic setting before getting admitted. They said that it helps them plan their activities and psychologically prepare for the procedure.

Another patient emphasised the fact that activities which should be avoided following surgery should be mentioned preoperatively. 'More explanation should be given on how to change our lifestyle after the surgery. Only when I was discharged I was told not to ride a bike or climb stairs. I barely make a living by riding my bike to deliver goods. My house is upstairs, and I cannot avoid climbing stairs. So, if the doctors informed me of these things before my surgery, I could have arranged someone to do my work and could stay elsewhere' (interviewee 27, component separation repair).

\section{Preoperative lack of information on vital complications}

Some patients voiced their anger and disappointment in the serious lack of vital details prior to surgery. 'When I woke up I noticed four tubes jutting out of me. I was not told that this will happen before the surgery. I felt very scared' (interviewee 2, anterior resection). 
Lack of question-answer sessions

Lack of question and answer sessions is expressed by the comment, 'I was not given enough time and information about the operation. So, I consulted a doctor in a private hospital to get more information about my operation' (interviewee 5, cholecystectomy).

\section{Failure in individualisation of consent}

Some were petrified that they will not want to undergo surgery on learning the negative outcomes of the surgical procedure. Therefore, they preferred not to know the complications. They wanted to deal with such complications by thinking 'the doctors tried their best'. This was apparent from the statement 'Knowing too many details about my surgery is not good. I'm afraid to hear them because it might make me say no to surgery. Even if the surgery goes wrong, I know the doctors tried their best and I will accept it' (interviewee 10, thyroidectomy). 'I know doctors are doing the best for us. Therefore, I do not wish to be educated about the adverse effects of the surgery' (interviewee 15, bariatric surgery).

However, some patients expected more details on the complications of surgery.

\section{Changes patients suggested for better understanding on what is being told to them}

All the patients did not report any issues with the language used. Use of other media such as handing out leaflets and booklets at the clinic was mentioned as a suggestion by a patient to aid them in being better informed about their surgical procedure. 'Give leaflets at the clinic or any books explaining the surgical procedure so that I can go home and read about it'. At the same time, she voiced her displeasure in seeing educational videos on surgery. 'I do not like to see educational videos or photos of the surgery. They have a lot of blood and makes me feel nauseated' (interviewee 1, mastectomy).

Some stated their disapproval in junior doctors taking ISC. They wanted senior consultants or the nurses to give out the details. 'The most junior doctor takes the consent, but we want the senior consultant to explain the procedure to us rather than a junior doctor. Consultants explain better' (interviewee 30, thyroidectomy). 'There is a busy atmosphere in the clinics. The nurses can tell us about the surgery rather than a doctor because they are easier to approach' (interviewee 28, thyroidectomy).

\section{DISCUSSION}

ISC, the heart of modern ethics, implies the inherent need of information to be delivered to the patient before the commencement of their surgical procedure. ${ }^{18}$ Sri Lankan patients' health beliefs and expectations deviate from the biomedical models applicable to other countries. ${ }^{19}$ Hence, the amount of details, content and the setting Sri Lankan patients wish to know about their surgery vary from the expectations of a patient from another country. ${ }^{19}$ Therefore, the need arises to understand what changes should be made in our setting to cater to the requirements of our patients.

\section{Finding the equilibrium between patient autonomy and disclosure of information}

All around the world the healthcare system is given a distinctive value. ${ }^{2021}$ Doctors were given the paternal role of deciding for the patients in the past. ${ }^{21} 22$ Western countries have adopted a more patient autonomous approach in consent. ${ }^{23}$ This concept was highlighted by the four principles in healthcare ethics by Beauchamp and Childress. ${ }^{8}$ Nevertheless, other countries also agree with the fact that if a patient refuses the full disclosure of information it is reflected as a part of patient autonomy. ${ }^{24}$

In our Sri Lankan society, we also encounter a mixture of opinions on information disclosure. Few patients depended on the surgeon to take the sole responsibility of decision-making and were open to accept any surgical outcome. This could be due to acceptance of the doctor's role to be a superior and dominant figure in the society. ${ }^{21} 22$ Social status and level of education could also be playing a role. $^{25}$ These patients did not consider ISC to be a mandatory procedure. According to Sri Lankan penal code, it is essential for the doctor to reveal 'all necessary facts' to the patient for them to make an intellectual decision. ${ }^{26} \mathrm{It}$ is the doctor who has the privilege of deciding what are considered necessary facts for the patient in such situations. Hall et alstated that in the USA some patients weigh risks against the benefits, while others base their choice of surgery on instinct. ${ }^{1}$ In addition, they also realised that some of the judgements of the patients were dependent on the institutional reputation and stature of the available surgeon. ${ }^{1}$ It is important to understand that individualism is a concept that intertwines around the traditional and cultural norms. ${ }^{27}$ It is highly subjective what one believes to be right or wrong. ${ }^{22}$ What one acknowledges to be correct could be unacceptable to another. Thus, it is mandatory to appreciate these variations and find the balance between patient autonomy and surgical recommendations. $^{22} 23$

Some patients felt that the delivery of details of their surgery was inadequate, yet they justified it by saying that the doctor was busy. Their view could be coming from a respectful place with regard to the medical profession. It is debatable as to what extent we ethically accommodate this idea as surgical professionals in our practice.

\section{ISC is not merely 'a formal procedure'}

Some of our patients thought they were obliged to sign the consent form regardless of their personal opinion. Some believed that ISC was a safety blanket for the surgeons to find a legal escape route in case of a detrimental outcome. Akkad et $a l^{28}$ reported that a majority $(70 \%)$ responded that they have a right not to sign the consent form. ${ }^{4}$ A British study reported an incidence of $88 \%$ stating that ISC was mainly for administrative and legal requirements. ${ }^{29}$ It is apparent that there were polarised views in the way patients saw the practicality of ISC. 
This highlights the need of patients to be educated on their role in ISC. Building the patients' trust and bridging the gap in current doctor-patient relationship is crucial. ${ }^{1}$

\section{Identify the essential components of the surgical consent}

In our study, one of the interviewees expressed his shock at discovering that he had several intubations made during surgery without his prior knowledge. In a study conducted on 200 participants in the UK, $76 \%$ of the patients agreed that just the right amount of information was delivered in ISC, while $20 \%$ said the content was too little. ${ }^{4}$ It is apparent that the content of the ISC is a worldwide issue. If the patient is likely to have major postoperative complications or any intensive care unit admissions, those details cannot be omitted. By withholding such vital data, the doctor is not only vulnerable to litigation but also violates medical ethics and patient's right for information. ${ }^{26}$ The ethical and legal goals must be met concurrently.

\section{Doctor need not wait until the ward admission to begin the consent-taking process}

The popular view among patients in the present study was that there was 'no' or 'scarce' amount of explanation on their surgery provided at the clinics before admission. Some did not understand why they were operated on; some did not know the postoperative need for radiotherapy. Majority were not aware of treatment options other than surgery. An interview-based British study emphasised the importance of learning if the patient 'wants' and 'is ready' to undergo surgery preoperatively. ${ }^{30}$ They discussed how some patients would disagree to surgery if certain details were disclosed at the time of taking consent. ${ }^{30}$

Consent taking ideally should begin from the moment the patient first encounters the doctor. ${ }^{31}$ In a medical consultation, the patient plays a dependent role. ${ }^{21}$ Having limited knowledge on medical procedures, they expect doctors to provide, not an extensive but a basic idea of the vital facts regarding their surgical treatment at the clinic. This is apparent by the patient's suggestion that the nurses should explain the details to them even if the doctor is busy.

It should be acknowledged that the patient numbers are high in a busy clinic at a major hospital. However, using methods like educational leaflets, counselling and patient education sessions by the nurses will reduce the burden on the surgeon's time in such a demanding environment. A systematic review (16 out of 21 studies) showed improvements in patient comprehension with written information provided preoperatively. A further 11 studies showed improvement with audio visual interventions. ${ }^{32}$ In our study, most interviewees advised against the use of audio clips for patient education.

One of the key elements we discovered during our study was that it is necessary to have a 'shared care' approach in order to deliver better patient care in a clinic setting. Patients suggested involving nurses in information delivery for consent. Implementation of this procedure will benefit not only the patient but also help breach the hierarchical gap between nurses and doctors in our healthcare system. This was supported by Swindale's statement that nurses interact more with the patients which allow them to recognise the patients' requirements and clarifications. ${ }^{33}$ Therefore, the role of nurses can be used to relieve preoperative anxiety in patients by delivering vital information regarding the surgery. ${ }^{33}$

\section{Spending time with the patient to improve patient comprehension}

Patients should be able to make sense of what is being done to their bodies. A surgical procedure done outside the framework of what is 'consented or authorised' by the patient is defined as 'assault'. ${ }^{26}$ Allocation of time for queries or clarification of doubts during clinics and the ward setting was brought up by patients during the interviews. Schenker et al stated the advancement of patient understanding of surgery by extended ISC discussions. ${ }^{32}$ In a UK-based study, Byrne et al concluded that unsatisfactory patient knowledge regarding their surgery was due to insufficient time and lack of communication skills of medical personnel. ${ }^{2}$

Giving patients an opportunity to clarify their issues is extremely important. ISC should not only be looked at as a legal obligation but also a symbol of moral medical practice. Even amidst busy schedules, the doctor-patient interaction should involve addressing the patients doubts and queries.

\section{Informed consent taking is a shared process}

In accordance with the bioethics and religious views of western countries, it is the patient's individual judgement that governs consent. ${ }^{18}$ In East Asia due to religious influences, there is a significant involvement of family members in decision-making. ${ }^{18}$ Also, in the Sri Lankan hospital care setting, dependence on family members in consenting was observed. ${ }^{34}$ Patients consider surgery as life-threatening. ${ }^{27}$ Since immediate family is considered to have the closest relationship to the patient, their views heavily influence the patient's decision.

In our study, we identified that the concept of surgery was stressful for patients. Presence of a family member helped reduce their stress level and provided a better counselling environment. This was corroborated by a study done in Taiwan, which depicted a marked influence by family members in the patient's decision-making for surgery. In this study, the expectation of family member participation in decision-making increased with the complexity of the surgery. ${ }^{27}$ Therefore, in our practice, a combined approach involving the surgeon, patient and family members should be adopted in the decision-making process while making the patient the focal point. $^{27}$

\section{Strengths and weaknesses of the study}

This is the first qualitative study conducted in Sri Lanka addressing the perceptions in surgical consent taking. 
Sample size of this study was limited to 30 ; therefore, generalisation of the results to the entire Sri Lankan population is not possible. But further multicentric quantitative or mixed research in a larger sample, based on the themes of the current research would help to develop national and regional guidelines on consent taking. Because of the time and resource constrains we were unable to explore the view of healthcare providers on informed consent. Since the face-to-face advertising method was used to recruit patients by healthcare providers, the patients might have been hesitant to decline participation. Hence, the positive response rate was excellent $(93.8 \%)$. However, in order to minimise this bias, care was taken to ensure that the primary interviewers were not attached to the same surgical units as the patients. And the patients were informed that the care given to them will not be compromised regardless of their participation or responses in the study. The high positive response rate in this study minimises self-selection bias. The study only explored patients undergoing major surgeries. However, this study can be used as a stepping stone to generate hypotheses, design future studies and guide to improve the insight on ISC.

\section{CONCLUSIONS}

Strictly adhering to western surgical consent-taking protocols might not be practically applicable in our setting due to the cultural and idealistic dissimilarities in South Asia. Patient autonomy should be made the focal point while involving the surgeons and family members in decision-making. Adequate time should be spent during the initial encounter at the clinic in providing patients' basic information on their surgery. A multidisciplinary contribution in counselling, use of leaflets and booklets may guide the patients to resolve their doubts in a busy surgical clinic or ward setting. Consent-taking process should be conducted in a conversational manner, rather than making it a forced process, in order to maintain a good doctor-patient relationship. In the future, further studies with combined qualitative and quantitative approaches using a nationally representative sample should be conducted. Also, the doctor's perspectives to overcome the identified problems in this study should be explored.

Contributors YM, ASKB and UMJES made substantial contribution to the conception and study design. YM and UMJES were the main contributor in data collection. YM, ASKB and UMJES were involved in refining the study design, critical analysis and drafting the manuscript. All authors read and approved the final manuscript.

Funding The authors have not declared a specific grant for this research from any funding agency in the public, commercial or not-for-profit sectors.

Competing interests None declared.

Patient consent for publication Not required.

Ethics approval Ethics Committee of National Hospital of Sri Lanka.

Provenance and peer review Not commissioned; externally peer reviewed.

Data sharing statement The translated interviews are available from the corresponding author on reasonable request.
Open access This is an open access article distributed in accordance with the Creative Commons Attribution Non Commercial (CC BY-NC 4.0) license, which permits others to distribute, remix, adapt, build upon this work non-commercially, and license their derivative works on different terms, provided the original work is properly cited, appropriate credit is given, any changes made indicated, and the use is non-commercial. See: http://creativecommons.org/licenses/by-nc/4.0/.

\section{REFERENCES}

1. Hall DE, Prochazka AV, Fink AS. Informed consent for clinical treatment. CMAJ 2012;184:533-40.

2. Byrne DJ, Napier A, Cuschieri A. How informed is signed consent? Br Med J 1988;296:839-40.

3. Grauberger J, Kerezoudis P, Choudhry AJ, et al. Allegations of failure to obtain informed consent in spinal surgery medical malpractice claims. JAMA Surg 2017;152:e170544-e44.

4. Cassileth BR, Zupkis RV, Sutton-Smith K, et al. Informed consent - why are its goals imperfectly realized? N Engl J Med 1980;302:896-900.

5. Wijerathne S, Kariyawasam D, Samarasinghe M, et al. Knowledge of parents regarding their child's surgical operation, 2009. https://sljs. sljol.info/articles/.

6. Best Practice protocols for clinical procedure safety: World Health Organization guidelines. http://www.who.int/surgery/publications/ immesc_best_practice/en/.

7. Hughes JC, Crepaz-Keay D, Emmett C, et al. The Montgomery ruling, individual values and shared decision-making in psychiatry. BJPsych Adv 2018;24:93-100.

8. Beauchamp TL. The 'four principles' approach to health care ethics. Principles of Health Care Ethics 2007;29:3-10.

9. Fan R. Self-determination vs. family-determination: two incommensurable principles of autonomy: a report from East Asia. Bioethics 1997;11:309-22.

10. Glasser B, Strauss A. The development of grounded theory. Chicago, IL: Alden, 1967.

11. Robinson OC. Sampling in interview-based qualitative research: $A$ theoretical and practical guide. Qual Res Psychol 2014;11:25-41.

12. Mason J. Qualitative researching. 2nd edition. London: Sage, 2002.

13. Trost JE. Statistically nonrepresentative stratified sampling: A sampling technique for qualitative studies. Qual Sociol 1986;9-54-7.

14. Birks M, Mills J. Grounded theory: A practical guide. Sage 2015.

15. Strauss A, Corbin JM. Basics of qualitative research: Grounded theory procedures and techniques: Sage Publications, Inc, 1990.

16. Strauss AL. Qualitative analysis for social scientists: Cambridge University Press, 1987. (Epub ahead of print: Jan 2010).

17. Guest G, Bunce A, Johnson L. How many interviews are enough? An experiment with data saturation and variability. Field methods 2006;18-59-82.

18. Meisel A, Kuczewski M. Legal and ethical myths about informed consent. Arch Intern Med 1996;156-2521-6.

19. Obeyesekere $\mathrm{G}$. The impact of Āyurvedic ideas on the culture and the individual in Sri Lanka. Asian medical systems: A comparative study 1976:201-26.

20. Quill TE, Brody H. Physician recommendations and patient autonomy: finding a balance between physician power and patient choice. Ann Intern Med 1996;125:763-9.

21. Savulescu J. Conscientious objection in medicine. BMJ 2006;332:294-7.

22. Murgic L, Hébert PC, Sovic S, et al. Paternalism and autonomy: views of patients and providers in a transitional (post-communist) country. BMC Med Ethics 2015;16:65.

23. Corrigan O. Empty ethics: the problem with informed consent. Sociol Health IIIn 2003;25:768-92.

24. Gillon R. Medical ethics: four principles plus attention to scope. BMJ 1994;309:184.

25. Mosley WH, Chen LC. AN analytical framework for the study of child survival in developing countries. Popul Dev Rev 1984;10:25-45.

26. Penal Code of Sri Lanka, 1883. Sections 340-342.

27. Lin ML, Kan WM, Chen CH. Patients' perceptions and expectations of family participation in the informed consent process of elective surgery in taiwan. Asian Nurs Res 2012;6:55-9.

28. Akkad A, Jackson C, Kenyon S, et al. Informed consent for elective and emergency surgery: questionnaire study. BJOG 2004;111:1133-8.

29. Satyanarayana Rao KH, Rao KS. Informed consent: an ethical obligation or legal compulsion? J Cutan Aesthet Surg 2008;1:33.

30. Meredith P. Patient participation in decision-making and consent to treatment: the case of general surgery. Sociol Health IIIn 1993;15:315-36. 
31. Anderson OA, Wearne IM. Informed consent for elective surgery-what is best practice? $J$ R Soc Med 2007;100:97-100.

32. Schenker Y, Fernandez A, Sudore R, et al. Interventions to improve patient comprehension in informed consent for medical and surgical procedures: a systematic review. Med Decis Making 2011;31:151-73.
33. Swindale JE. The nurse's role in giving pre-operative information to reduce anxiety in patients admitted to hospital for elective minor surgery. J Adv Nurs 1989;14:899-905.

34. Gunatillaka KAN. "Patient centered care, high tech with a soft touch". Journal of the Ceylon College of Physicians 2016;47:3. 